\title{
HOW DONATED BLOOD IS TESTED: AN INTERVIEW WITH CANADIAN BLOOD SERVICES
}

\section{Eureka Team}

Sherwin Dziwenka, Regional Manager of Western Donor Testing, outlines the testing protocol undertaken by Canadian Blood Services when screening the various blood products collected.

\section{i) Who is responsible for testing of blood products collected by Canadian Blood Services?}

Western Consolidated Donor Testing Laboratory tests donor samples for all mandatory and supplemental tests from British Columbia, Alberta, Saskatchewan, Manitoba and the Yukon Territories.

\section{ii) For which diseases are the collected blood products tested?}

The collected blood is tested for the following infectious diseases: Syphilis, Human Immunodeficiency Virus 1 and 2, Hepatitis B and C, Human T-Cell Lymphotropic Virus I and II, and West Nile Virus. These tests are mandatory and must be performed on every CBS donor sample.

There are 2 additional tests that may or may not be performed, which are dependant on:

- specific recipient requirements, which may result in the collected blood being additionally tested for Cytomegalovirus;

- and donor history, which may result in the collected blood being additionally tested for Chagas.

\section{iii) How is testing on collected blood products} undertaken?

Testing for the purpose of blood donor screening must meet regulatory requirements and quality assurance expectations. Testing is required for each donation and must use a concurrent drawn sample.

Serological tests are initially performed singly; nonreactive results are interpreted as negative, allowing release of the component for transfusion. Reactive results are repeated in duplicate: if both repeat results are nonreactive, then the result is interpreted as negative. If one or both repeat results are reactive, then the test result is considered "repeat reactive" and the associated blood donation cannot be used for transfusion. Supplemental tests are performed on repeat-reactive samples primarily for donor notification and counseling purposes.

NAT testing begins with a number of blood samples being "pooled" together in the laboratory. If the result of a pool of samples is negative, all samples in the pool are negative. If the pool is positive, then the samples are tested in smaller pools and then individually until the actual positive sample is identified.

Questions written by the Eureka Editorial Team with answers provided by Sherwin Dziwenka, Regional Manager of Western Donor Testing for Canadian Blood Services.

\section{Eureka}

Volume 2, Number 1 (2011)

With each unit of blood, platelets or plasma collected there are 5 samples (4 plasma samples and 1 serum sample) collected and sent to laboratory for testing.

First plasma sample is sent to the Blood Group Serology Laboratory to perform:

- $\mathrm{ABO}$ Grouping

- Rh Type

- RBC Antibody Screen

- Syphilis test

- Cytomegalovirus test, when applicable

Second plasma sample goes to the Nucleic Acid Testing Laboratory to perform:

- West Nile Virus RNA test

Third plasma sample goes again to the Nucleic Acid Testing Laboratory to perform:

- Hepatitis C Virus RNA test

- Human Immunodeficiency Virus -1 RNA test

Fourth plasma sample is called "Retention Sample" and is used for any confirmatory or Chagas testing if required. Chagas testing is only performed on samples for a donor that answered yes to one or more of the 3 Chagas risk questions during donor registration.

The one serum sample goes to Transmissible Disease Laboratory to perform:

- Antibody to Human Immunodeficiency Virus Type 1 and 2 test

- Hepatitis B Surface Antigen test

- Antibody to Hepatitis C Virus test

- Antibody to Human T-Cell Lymphotropic Virus Type I/II test

- Antibody to Hepatitis B Virus Core Antigen test

\section{iv) Please explain in detail one particular test.}

ABO grouping

The determination of an $\mathrm{ABO}$ blood group is done by demonstrating the presence or absence of antigens $A$ and/or $B$ on the surface of human red blood cells and by detecting the presence or absence of anti-A and/or anti-B antibodies in the plasma. If an individual has an antigen on the surface of his/her red blood cell then they don't have the corresponding antibody in the plasma. It is therefore appropriate to identify the erythrocyte antigens using known anti-A and anti-B, then to confirm the results by verifying the presence of the corresponding antibodies in the plasma from the test blood using known red blood cells A1 and B, also known as reverse grouping. There is also testing of red blood cells with Anti-A,B. It is an additional check for the presence of $A$ and $B$ antigen as well as $a$ 
facilitator in recognition of certain weak subgroups. The test is based on the principle of agglutination and pattern recognition. When red blood cells have the antigen on their surface, agglutination will occur with the reagent containing the corresponding antibody. Agglutination with an antibody indicates the presence of the specific antigen. If there is no agglutination it means that the red blood cells lack the antigen. This is how you determine if somebody is blood group: $A$ ( $A$ antigen is present), $B$ ( $B$ antigen is present), $A B$ (both $A$ and $B$ antigens are present) or $O$ (neither $A$ nor $B$ antigen is present).

\section{v) How long, on average, does the entire testing process} take?

The entire testing process takes approximately 12 hours from the time sample arrives to the Sample Management Lab till the time all the results are ready.

vi) If blood products collected from an individual test positive on a particular test, what is done with these blood products?

If this positive result is for a transmissible disease (eg HIV), the blood products are destroyed by incineration.

\section{vii) What does a "false positive" result mean? What is done with blood products that test positive falsely?}

The tests performed by Canadian Blood Services are extremely sensitive and they sometimes produce "false positive "results. This occurs in a small number of donors when a substance or substances in their healthy blood trigger a reaction. To determine if reactive results are truly positive, all units that produce repeat reactive results with screening tests are subjected to additional confirmatory testing. If confirmatory testing produces a negative result, the unit is considered a "false positive". A negative result in confirmatory testing is reassuring news for the healthy donor.

Nevertheless, no unit that produces a repeat reactive result with a screening test is ever transfused. Canadian Blood Services takes extra precautionary measures to ensure safety. It destroys any blood unit that tests "false positive" and maintains records so that these individuals are not accepted for blood donations in the future.

viii) Often the term window period is referred to during testing of blood products. What does this term mean?

Most traditional screening tests require the presence of antibody to trigger a positive test reaction. The period of time between initial infection and detection of antibodies is called the "window period".

\section{ix) What steps are undertaken to maximize safety for} recipients, despite the existence of a 'window period'?

Selection of eligible blood donors is a critically important step to maximize the safety and integrity of the blood supply. Selection of healthy donors by interviews and limited physical examination represents the first protective layer against infectious disease transmission.

Deferral of donors with certain past or current medical conditions, donors who engage in activities known to increase risk to the blood supply, immigration background, travel histories or specific physical findings minimizes the risk of collecting blood, plasma or platelets contaminated with transmissible agent.

In addition to screening out individuals whose health or high risk activities may put recipients at risk, stringent laboratory tests for infectious diseases are conducted on each unit of blood, plasma or platelets.

x) What is nucleic acid testing (NAT)? Why is it being increasingly used in Canadian Blood Services' testing procedures? What are its benefits versus traditional antibody/antigen testing?

Nucleic Acid Testing (NAT) detects the presence of viral genetic material in donor samples. Detection of viral RNA or DNA involves three steps: extraction, amplification and detection. Extraction occurs first and is followed by capture of viral RNA or DNA onto a capture molecule that is immobilized on a solid phase or onto magnetic particles in a solution. Once immobilized, the impurities are removed by a series of wash steps. Prior to amplification, viral RNA must be converted to DNA; this is accomplished by the enzyme, reverse transcriptase. Amplification of the DNA then can occur through multiple intermediates. Detection of the amplified product occurs by capture of the amplified DNA on nitrocellulose or by enzyme immunoassay or chemiluminescence.

NAT works by detecting the low levels of viral genetic material present when an infection occurs but before the body begins producing antibodies in response to a virus.

NAT significantly reduces the "window period". It allows for earlier detection of the infection and thus further decreases the possibility of transmission via transfusion. That is why it is increasingly used by CBS.

NAT is used in addition to antibody tests. Some individuals infected with Hepatitis $\mathrm{C}$ or HIV may be NAT - negative if the amount of virus present has fallen below detectable levels. In such cases antibodies to the virus would still be detected by current more traditional tests for Hepatitis C or HIV.

\section{xi) Whose blood is tested? Are blood products from} individuals who have previously donated also tested?

No matter how many previous donations, each unit of blood, plasma or platelets collected is tested as outlined above. Every donation has to have all the mandatory tests completed before any products made from that donation can be released for use. If any of the mandatory tests can't be completed on a given donation all the products from that donation are destroyed.

xii) How important are these testing procedures to the safety of Canada's blood supply?

It is possible for infectious agents to circulate in the bloodstream for weeks, months, or even years without causing any symptoms. Infected persons may unknowingly present as blood donors, and their donations may be accepted. In these cases, the next barrier to maintain the safety of the blood supply is effective blood donor 
screening tests. Fortunately, sensitive tests for selection of donors are available and their use is mandated whenever blood is collected for the purpose of transfusion.

xiii) Is there any piece of information you believe our reader's should know about the testing of blood products at Canadian Blood Services?

Safety is the paramount concern of Canadian Blood Services. Because blood is a biopharmaceutical product, it must be manufactured within very strict regulatory standards using Good Manufacturing Practices. As a result of Canadian Blood Services commitment to meeting national and international safety standards, Canada's blood supply is recognized as one of the safest in the world. Donor Testing has a very important role within the organization to ensure the safety of blood supply in Canada. We use the most advanced "state of the art" methods of testing blood for the presence of infectious diseases and blood groups.

There is a constant need for blood. To book your appointment please call 18882 DONATE.

For more information on blood awareness, contact the University of Alberta Blood Awareness and Donation Students Association (BADSA) at

http://www.ualberta.ca/ badsa/

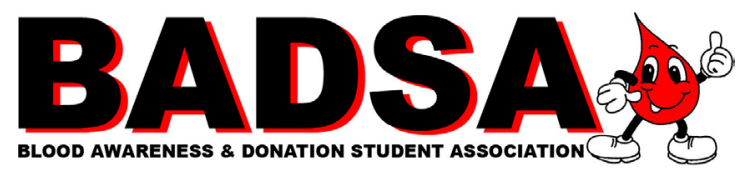

\section{Discover Undergraduate Research}

\section{Do you want to:}

- make your undergraduate degree

stand out?

- develop connections with

faculty?

- investigate topics you're

passionate about?

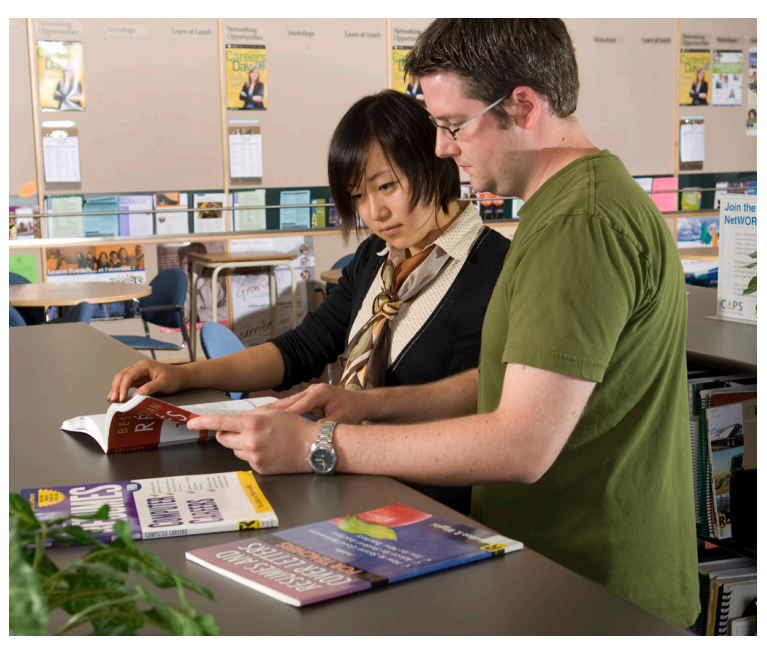

Getting involved in undergraduate research is a great way to do these things and more. Visit CAPS and get ideas about how to get involved. 